\title{
Comparison of Growth Rate of Electromagnetic Waves in Pre-bunched Cerenkov Free Electron Laser and Free Electron Laser
}

\author{
Anuradha Bhasin and Bhupesh Bhatia \\ Department of Electronics and Communication, Northern India Engineering College, FC-26, Shastri Park, \\ G.G.S.I.P. University, Delhi-110053, India.
}

\begin{abstract}
An electron beam passing through a slow wave structure is unstable to an electromagnetic perturbation whose phase velocity equals the velocity of the beam. This phenomenon of cerenkov emission is the basis of all slow wave devices. The increase in the growth rate by using a pre-bunched electron beam in a Cerenkov free electron laser (CFEL) and Free electron laser (FEL) has been studied. We have compared the increase in growth rate with the increase in modulation index calculated at experimentally known CFEL and FEL parameters.
\end{abstract}

\section{Keywords}

Free electron laser, Cerenkov free electron laser, Pre-bunched electron beam.

\section{INTRODUCTION}

Cerenkov free electron laser (CFEL) is the widely used source of broad- band, high power microwave generation at short wavelengths. In this device, an electron beam passing through wave structure resonantly interacts with wave whose phase velocity equals the drift velocity of electrons and the wave grows at the expense of energy of the beam. Since the electron velocity cannot exceed the velocity of light, a slow wave structure is needed to slow down the phase velocity of electromagnetic modes. In case of Cerenkov free electron laser (CFEL) [1] which employs a slow wave medium to slow down the phase velocity of transverse electric (TE) or transverse magnetic (TM) modes to less than c, the velocity of light so that they can be excited by a moderately relativistic electron beam by the process of cerenkov emission. A Cerenkov free electron laser generally employs two kinds of slow wave structures: (i) A dielectric whose dielectric constant is $|\varepsilon|>1$ reduces the phase velocity of the radiation below c. A moderately relativistic electron beam can excite the electromagnetic radiation by cerenkov emission, (ii) A plasma lining have a dielectric constant $\varepsilon=1-\frac{\omega_{p}^{2}}{\omega^{2}}$ can act as a slowing down medium for $\omega_{p} \gg \omega$ so that $|\varepsilon| \gg>1$ (where $\omega_{p}$ is the electron plasma frequency and $\omega$ is the radiation frequency).

A CFEL consisting of two dielectrically lined parallel plates driven by dense moderately relativistic electron beam has been studied and reported to produce coherent high power radiation from 375 micrometer to $1 \mathrm{~mm}$ wavelengths [2-4].
More recently, a lot of research work has been carried out in studying the free electron laser [5-12] by pre-bunched electron beams. A high power microwave free electron laser experiment has been performed using pre-bunched electron beam of $35 \mathrm{Mev}$ [6]. Here when the electron beam is prebunched at a frequency close to an eigen frequency of the cavity, the oscillation build process is speed up and the radiation build time is shortened significantly. Free electron maser experiment with a pre-bunched electron beam has been demonstrated at Tel Aviv University [7]. In this case, they utilize a $1.0 \mathrm{~A}$ current pre-bunched electron beam obtained from a microwave tube. The electron beam is bunched at $4.87 \mathrm{GHz}$ frequency and is subsequently accelerated to 70 $\mathrm{KeV}$. The bunched beam is injected into a planar wiggler $\left(\mathrm{B}_{\mathrm{W}}\right.$ $=300$ gauss, $\lambda_{W}=4.4 \mathrm{~cm}$, where $\mathrm{B}_{\mathrm{W}}$ is the wiggler field and $\lambda_{W}$ is the wiggler wavelength) constructed in a Halbach configuration with 17 periods. A theoretical model for gain and efficiency enhancement in a FEL using pre-bunched electron beam has been developed and studied by Beniwal et al. [11]. Sharma and Bhasin have studied the gain and efficiency enhancement in a slow wave FEL using prebunched electron beam in a dielectric loaded waveguide [12]. They have found that the growth rate and gain of a slow wave FEL increase with the increase in modulation index and is maximum when the pre-bunched beam velocity is comparable to the phase velocity of the radiation wave.

In this paper, we develop a theoretical model of a prebunched CFEL and present the analytical analysis for the excitation of electromagnetic waves by a pre-bunched electron beam in a CFEL. We compare the increase in growth rate with the increase in the modulation index for pre-bunched CFEL with a pre-bunched FEL. The growth rate has been calculated at experimentally known CFEL and FEL parameters.

\section{INSTABILITY ANALYSIS}

Consider a dielectric loaded waveguide of effective permittivity $\mathcal{E}_{1}$. A pre-bunched relativistic electron beam of density $\mathrm{n}_{\mathrm{b} 0}$, velocity $v_{b}$, relativistic gamma factor $\gamma=1+\frac{e V_{b}}{m c^{2}}\left(1+\Delta \sin \omega_{0} \tau\right) \approx \gamma_{0}\left(1+\Delta \sin \omega_{0} \tau\right) \quad$ [where $\Delta$ is the modulation index (its value lie from 0 to 1 ), $\mathrm{mc}^{2}$ is the rest mass energy of the electrons, $\mathrm{e}$ is the electronic charge, $\omega_{\mathrm{O}}\left(\approx k_{z \mathrm{O}} v_{b}\right)$ and $k_{z 0}$ are the modulation frequency and wave number of the pre-bunched electron 
beam], respectively propagates through the waveguide (cf. Fig. 1).

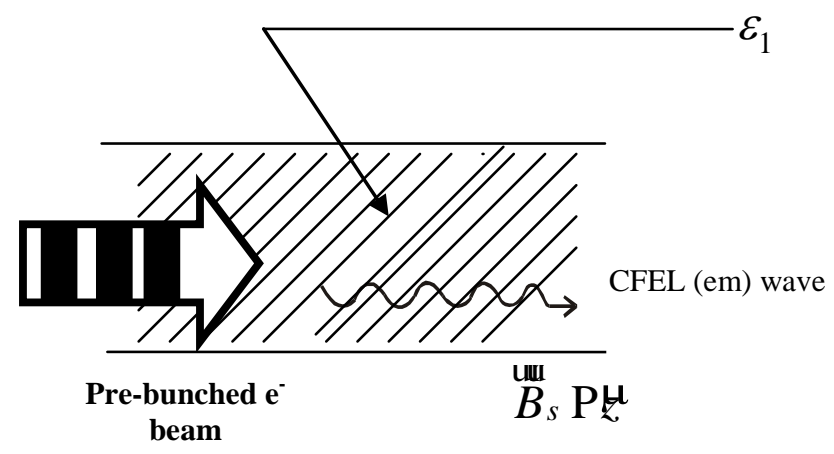

Fig1: A schematic diagram of the CFEL

An electromagnetic signal $E_{1}$ is also present in the interaction region.

$$
\operatorname{un}_{E_{1}=E_{0}} e^{-i\left(\omega_{1} t-k_{1} \cdot \bar{x}\right)},
$$

$\underset{B_{1}}{\mathbf{u}}=\frac{c}{\omega_{1}} \underset{1}{\mathbf{u r}} \underset{E_{1}}{\mathbf{u r}}$,

where ${\stackrel{\mathbf{u}}{E_{0}}}_{0}$ and $\stackrel{\mathbf{u}}{k}_{1}$ lie in the $\mathrm{x}-\mathrm{z}$ plane, i.e., $\frac{\partial}{\partial y}=i k_{y}=0$

. The response of the beam electrons to the signal is governed by the relativistic equation of motion

$$
\frac{\partial}{\partial t}(\gamma v)+v \cdot \nabla(\gamma v)=-\frac{e}{m} \underset{c}{\mathbf{r}}\left(E+\frac{1}{c} v \times B\right) .
$$

Velocity components in the $\mathrm{x}$ and $\mathrm{z}$ directions are given by

$$
\begin{aligned}
& v_{x 1}=\frac{e}{i m \gamma\left(\omega_{1}-k_{z} v_{b}\right)}\left[E_{x 1}-\frac{k_{z} v_{b} E_{x 1}}{\omega_{1}}+\frac{k_{x 1} v_{b} E_{z 1}}{\omega_{1}}\right] . \\
& v_{z 1}=\frac{e E_{z 1}}{i m\left(\omega_{1}-k_{z} v_{b}\right) \gamma^{3}} .
\end{aligned}
$$

On linearizing and solving equation of continuity, we obtain density perturbation

$$
n_{1}=n_{b 0} \frac{k 1 . v_{1}}{\left(\omega_{1}-k_{z} v_{b}\right)} .
$$

Using the value of $\mathrm{v}_{\mathrm{x} 1}$ and $\mathrm{v}_{\mathrm{z} 1}$ from equations (5) and (6) in equation (7), we get

$n_{1}=\frac{e n_{b 0}}{i m\left(\omega_{1}-k_{z} v_{b}\right)^{2}}\left[\frac{E_{x 1} k_{x 1}}{\gamma}\left(1-\frac{k_{z} v_{b}}{\omega_{1}}\right)+\frac{k_{x 1}^{2} v_{b} E_{z 1}}{\gamma \omega_{1}}+\frac{k_{z 1} E_{z 1}}{\gamma^{3}}\right]$.

The perturbed current density is given by

$$
J_{1}=-n_{b 0} e v_{1}-n_{1} e v_{b} \text {. }
$$

Substituting the values of $v_{1}$ and $\mathrm{n}_{1}$ from equations (5), (6) in equation (8), and keeping the value in the wave equation, we obtain

$\left(k_{z 1}^{2}-\frac{\omega_{1}^{2}}{c^{2}} \varepsilon+\frac{\omega_{p b}^{2}}{\gamma c^{2}}\right) E_{x 1}=\left(k_{x 1} k_{z}-\frac{\omega_{p b}^{2}}{\gamma c} \frac{k_{x 1} v_{b}}{\left(\omega_{1}-k_{z} v_{b}\right)}\right) E_{z 1}$,

where $\omega_{p b}^{2}=\frac{4 \pi n_{b o}}{m} e^{2}$.

Equation (9) can be further rearranged by taking $\omega_{p b}{ }^{2}$ terms to the right hand side and retaining only those terms which have a resonance denominator $\left(\omega_{1}-k_{z} v_{b}\right)^{2}$, we get

$$
\left(\omega_{1}^{2}-\frac{k_{1}^{2} c^{2}}{\varepsilon}\right)\left(\omega_{1}-k_{z} v_{b}\right)^{2}=\frac{\omega_{p b}^{2}}{\gamma^{3} \varepsilon}\left(\omega_{1}^{2}+k_{x 1}^{2} v_{b}^{2} \gamma^{2}\right) .
$$

The two factors on the left-hand side of equation (10) when equated to zero $\omega_{1}-\frac{k_{1} c}{\sqrt{\varepsilon}}=0, \omega_{1}-k_{z} v_{b}=0$, give radiation and beam modes, respectively. To determine the growth rate of the CFEL instability, we use the first order perturbation techniques .In the presence of the right hand side terms (i.e., $\left.n_{b 0} \neq 0\right)$, we assume that the eigen functions are not modified but their eigen value are. We expand $\omega_{1}$ as

$\omega_{1}=\omega_{1 r}+\delta=k_{z} v_{b}+\delta=k_{z 0} v_{b}+\delta$,

where $\delta$ is the small frequency mismatch and $\omega_{1 r}=\frac{k_{1} c}{\sqrt{\varepsilon}}$.

On further solving equation (12) we obtain

$\delta=\left[\frac{\omega_{p b}^{2}\left(\omega_{1 r}^{2}+k_{x 1}^{2} v_{b}^{2} \gamma^{2}\right)}{2 \omega_{1 r} \gamma^{3} \varepsilon}\right]^{1 / 3} e^{i \frac{2 n \pi}{3}}, n=0,1,2,3, \ldots \ldots$.

Hence the growth rate, i.e., the imaginary part of $\delta$ is given as

$\Gamma=\left[\frac{\omega_{p b}^{2}\left(\omega_{1 r}^{2}+k_{x 1}^{2} v_{b}^{2} \gamma_{0}^{2}\right)}{2 \omega_{1 r} \gamma^{3} \varepsilon}\right]^{1 / 3} \frac{\sqrt{3}}{2}$

where $\gamma=\gamma_{0}\left(1+\Delta \sin \omega_{0} \tau\right)$.

For maximum gain it is assumed that all electrons are bunched in the decelerating zone, i.e., $\omega_{0} \tau=-\pi / 2$. This gives $\gamma=\gamma_{0}(1-\Delta)$, where $\Delta$ is the modulation index, its value lies between 0 to 1 and $\Delta \neq 1$.

\section{RESULTS AND DISCUSSIONS}

In the numerical calculations we have used typical parameters of Cerenkov free electron laser (CFEL) [1]. For the comparative study we have also used parameters of free electron maser experiment with a pre-bunched electron beam , (e.g., beam energy $=0.08 \mathrm{MeV}$ and beam current $\mathrm{I}_{\mathrm{b}}=1.0 \mathrm{~A}$ ) and other parameters are same as CFEL. The corresponding parameters are given in the Table 1. 
Table 1: Parameters for Fig.2. $\mathrm{E}_{\mathrm{b}}, \mathrm{I}_{\mathrm{b}}, \mathrm{A}_{\mathrm{b}}$ are the electron beam energy, beam current and beam cross-section respectively. $\omega_{0}$ and $\varepsilon$ are the modulation frequency of the pre-bunched beam and effective permittivity.

\begin{tabular}{lll}
\hline Notations & $\begin{array}{l}\text { Values } \\
(\text { CFEL })\end{array}$ & $\begin{array}{l}\text { Values } \\
(\text { FEL })\end{array}$ \\
\hline $\mathrm{E}_{\mathrm{b}}(\mathrm{MeV})$ & 1.30 & 0.08 \\
$\mathrm{I}_{\mathrm{b}}(\mathrm{A})$ & 900 & 1.0 \\
$\mathrm{~A}_{\mathrm{b}}\left(\mathrm{cm}^{2}\right)$ & 0.196 & 0.196 \\
$\mathrm{w}_{0}(\mathrm{GHz})$ & 5.00 & 5.00 \\
$\boldsymbol{E}$ & 1.7 & 1.7 \\
$\mathrm{~K}_{1}\left(\mathrm{~cm}^{-1}\right)$ & 1.3 & 1.3 \\
\hline
\end{tabular}

In Fig. 2, we have plotted the variation of the growth $\Gamma$ (in $\mathrm{rad} / \mathrm{sec}$ ) as a function of modulation index $\Delta$ wen the phase of the pre-bunched beam is $-\pi / 2$, i.e., when the electron beam is in the decelerating zone, (a) plot for CFEL parameters and (b) plot for FEL parameters. From Fig. 2, it can be seen that the growth rate increases with the modulation index (in both cases), when $\Delta \approx 0.85$ and beyond this value of modulation index, i.e., when $\Delta$ increases from 0.85 to 0.98 , the growth rate increases by a factor of 9 for CFEL parameters and by 10 for FEL parameters. The growth rate of the CFEL instability scales as one third power of the beam current and minus one third power of the effective permittivity [cf. eq. (12)].

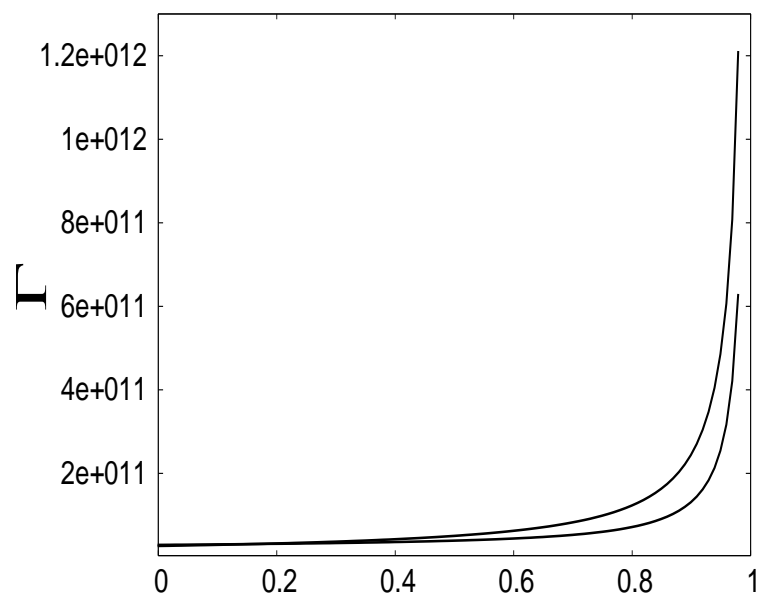

Modulation-index $\Delta$

Fig 2: Growth rate $\Gamma$ (in $\mathrm{rad} / \mathrm{sec}$ ) as a function of modulation index $\Delta$ for (a) CFEL parameters with $E_{b}=$ $1.30 \mathrm{MeV}, I_{b}=900.0 \mathrm{~A}$ and for $\sin \omega_{0} \tau=-1$, (b) FEL

parameters with $E_{b}=0.08 \mathrm{MeV}, I_{b}=1.0 \mathrm{~A}$ and for sin $\omega_{0} \tau=-1$. Increase in growth rate is more for case (b) i.e., with FEL parameters.

\section{CONCLUSION}

In conclusion, we can say that the beam pre-bunching on Cerenkov free electron laser offers considerable enhancement in growth rate and hence enhancement in efficiency when the beam electrons are in the retarding zone and the phase velocity of the radiation is comparable to the pre-bunched beam velocity. The comparative study shows that the increase in growth rate of the electromagnetic wave is more in FEL than in CFEL, as the modulation index increases from 0.85 to 0.98 .

Pre- bunched CFEL scheme may find an important application in the generation of high frequency radiations and as compared to FELs, CFELs are simple to design and tune. In addition to this, it is seen that beam pre-bunching in CFEL and FEL can reduce the requirement for beam energy to generate high frequency radiations.

\section{REFERENCES}

[1] J. Walsh, B. Johnson, G. Dattolii and A. Renieri. Undulator and Cerenkov free electron lasers: a preliminary comparison, Phys. Rev. Lett., vol. 53, pp. 779-782, 1984.

[2] B. Johnson and J. Walsh, "A Cerenkov infrared laser," Nucl. Instr. Methods Phys. Res., vol. A237, pp. 239-243, 1985.

[3] E. P. Garate, C. Shaughnessy, J. Walsh and S. Moustaizis, "Cerenkov free-electron laser operation at far-infrared to sub-millimeter wavelengths," in Proc. $8^{\text {th }}$ Int. Free-Electron Laser Conf., Glasgow, Scotland, Sept. 1986.

[4] E. P. Garate, R. Cook, P. Heim, R. Layman, and J. Walsh, "Cerenkov maser operation at lower $\mathrm{mm}$ wavelengths," J. Appl. Phys., vol. 58, pp.627-632, 1985.

[5] J. Krall and Y.Y. Lau, "Modulation of an intense beam by an external microwave source: theory and simulation," Appl. Phys. lett., vol. 52, pp. 431-433, 1988.

[6] Yukio Shibata, Kimihiro Ishi, Shuichi Ono et al., "Broadband free electron laser by the use of pre-bunched electron beam," Phys. Rev. Lett., vol. 78, pp. 2740-2743, 1997.

[7] M. Cohen, A. Kugel, D. Chairman, M. Arbel et al., "Free electron maser experiment with a pre-bunched beam," Nucl. Instr. and Methods in Phys. Res., vol. A358, pp. 82-85, 1995.

[8] Kazuyoshi Saito, Ken Takayama, Toshiyuki Ozaki et al., "X-band prebunched FEL amplifier," Nucl. Instr. and Methods in Phys. Res., vol. A375, pp. 237-240, 1996.

[9] H. P. Freund, P. G. O'Shea, J. Neumann, "Simulation of pre-bunching in free-electron lasers," Nucl. Instr. and Methods in Phys. Res., vol. A507, pp. 400-403, 2003.

[10] J. Gardelle, J. Labrouche, G. Marchese, J. L. Rullier and D.Villate and J. T. Donohue, "Analysis of the beam bunching produced by a free electron laser," Phys. Plasmas, vol. 3, pp. 4197-4206, 1996.

[11] V. Beniwal, S.C. Sharma, and M. K. Sharma, "Effect of beam premodulation on gain and efficiency in a free electron laser," Phys. Plasmas, vol. 11, pp. 5716-5722, 2004.

[12] Anuradha Bhasin and Suresh C. Sharma. Effect of Beam Premodulation and Guide Magnetic Fields on Slow Wave Free Electron Laser: Nonlocal Theory, IEEE Journal of Quantum Electronics, Vol. 45, No. 9, 11291132, 2009. 